\title{
(6) OPEN ACCESS \\ Effect of subthalamic nucleus deep brain stimulation on dual-task cognitive and motor performance in isolated dystonia
}

\author{
Kelly A Mills, ${ }^{1,2}$ Leslie C Markun, ${ }^{1}$ Marta San Luciano, ${ }^{1}$ Rami Rizk, $^{3}$ I Elaine Allen, ${ }^{4}$ \\ Caroline A Racine, ${ }^{5}$ Philip A Starr, ${ }^{5}$ Jay L Alberts, ${ }^{3}$ Jill L Ostrem ${ }^{1,2}$
}

- Additional material is published online only. To view please visit the journal online (http://dx.doi.org/10.1136/ jnnp-2014-307942).

${ }^{1}$ Department of Neurology, University of California, San Francisco, California, USA ${ }^{2}$ Parkinson's Disease Research, Education, and Clinical Center, San Francisco Veterans Affairs Medical Center, San Francisco, California, USA

${ }^{3}$ Department of Biomedical Engineering, Cleveland Clinic, Cleveland, Ohio, USA ${ }^{4}$ Department of Epidemiology \& Biostatistics, University of California San Francisco, San Francisco, California, USA ${ }^{5}$ Department of Neurosurgery, University of California San Francisco, San Francisco, California, USA

\section{Correspondence to} Dr Jill Ostrem, Surgical Movement Disorders Center, University of California San Francisco, 1635 Divisadero Street, Suite 520, UCSF Box 1838, San Francisco, CA 94143-1838, USA; jill.ostrem@ucsf.edu

Received 21 February 2014 Revised 27 May 2014 Accepted 14 June 2014 Published Online First 10 July 2014

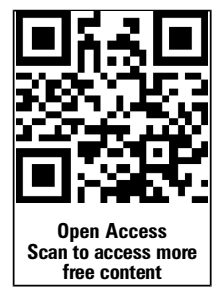

CrossMark

To cite: Mills KA, Markun LC, San Luciano M, et al. I Neurol Neurosurg Psychiatry 2015;86: 404-409.

\section{ABSTRACT}

Objective Subthalamic nucleus (STN) deep brain stimulation (DBS) can improve motor complications of Parkinson's disease (PD) but may worsen specific cognitive functions. The effect of STN DBS on cognitive function in dystonia patients is less clear. Previous reports indicate that bilateral STN stimulation in patients with PD amplifies the decrement in cognitive-motor dual-task performance seen when moving from a singletask to dual-task paradigm. We aimed to determine if the effect of bilateral STN DBS on dual-task performance in isolated patients with dystonia, who have less cognitive impairment and no dementia, is similar to that seen in PD.

Methods Eight isolated predominantly cervical patients with dystonia treated with bilateral STN DBS, with average dystonia duration of 10.5 years and Montreal Cognitive Assessment score of 26.5 , completed working memory (n-back) and motor (forced-maintenance) tests under single-task and dual-task conditions while on and off DBS.

Results A multivariate, repeated-measures analysis of variance showed no effect of stimulation status (On vs Off) on working memory ( $F=0.75, p=0.39$ ) or motor function $(F=0.22, p=0.69)$ when performed under single-task conditions, though as working memory task difficulty increased, stimulation disrupted the accuracy of force-tracking. There was a very small worsening in working memory performance $(F=9.14, p=0.019)$ when moving from single-task to dual-tasks when using the 'dual-task loss' analysis.

Conclusions This study suggests the effect of STN DBS on working memory and attention may be much less consequential in patients with dystonia than has been reported in PD.

\section{INTRODUCTION}

Dystonia is a movement disorder in which sustained or intermittent muscle contractions cause abnormal, often repetitive, movements, postures or both which may be patterned, twisting and/or tremulous. ${ }^{1}$ Current evidence points to a pathophysiology involving broad network-based changes of the basal ganglia-thalamic-cortical circuit ${ }^{2}$ without neurodegeneration. Cognitive function was initially described as relatively normal in patients with isolated dystonia, ${ }^{3}{ }^{4}$ but mild deficits in executive functions are described. ${ }^{5} 6$ However, the degree of disability from cognitive impairment is thought to be minimal in comparison to healthy controls. $^{7}$

Deep brain stimulation (DBS) in the globus pallidus internus (GPi) is effective for treatment of isolated idiopathic or inherited dystonia, ${ }^{8-10}$ including generalised $^{9}$ and segmental forms. ${ }^{11}{ }^{12}$ The subthalamic nucleus (STN) has been explored as an alternative target for DBS in primarily cervical dystonia ${ }^{13-15}$ following the observed side effects of GPi stimulation, including micrographia, ${ }^{12}$ bradykinetic gait, ${ }^{16}$ parkinsonism ${ }^{17}$ and limb bradykinesia. ${ }^{18}$ Alternative stimulation targets, such as the thalamic-subthalamic area, are also being explored to treat specific dystonic symptoms such as dystonic head tremor. ${ }^{19}$

Concern has been raised over the possible cognitive adverse effects after STN DBS in the treatment of movement disorders. A meta-analysis describing cognitive outcomes in STN DBS for Parkinson's disease (PD) showed mild but measurable impairment in executive function, verbal learning and memory, ${ }^{20}$ with verbal fluency ${ }^{21}$ consistently worsening to a greater extent following STN DBS than with GPi DBS. ${ }^{22}$

Declines in working memory and attention may contribute to an increased fall rate secondary to postural instability ${ }^{23}$ and gait variability. ${ }^{24}$ Some studies in patients with PD have shown that STN DBS worsens attention or working memory, ${ }^{25} 26$ which raises concerns that cognitive adverse effects of STN DBS could diminish the value of motoric improvements. It is thought that a dual-task paradigm may more closely simulate attention and working memory requirements in a real-world setting. ${ }^{27}$ In such a paradigm, it was shown that STN stimulation worsened dual-task function in patients with $\mathrm{PD}$, and that this loss of performance in both tasks is exaggerated with increasing task difficulty. ${ }^{28}$

The effect of DBS on cognitive performance in patients with dystonia is even less well described. Patients with GPi DBS for dystonia showed preserved cognitive function. ${ }^{39} 29$ No declines in performance on a full neuropsychological battery were observed in nine patients with STN DBS for dystonia, though the study was not powered to evaluate cognitive function as a primary outcome. ${ }^{13}$

Considering the potential use of STN as a target for DBS in the treatment of dystonia, and concerns regarding stimulation-induced cognitive changes in STN DBS for PD, we aimed to investigate the 
effects of STN DBS on cognition in dystonia using a cognitivemotor dual-task paradigm that has shown subtle stimulation-induced changes in previous studies. ${ }^{28}$ In the absence of an underlying pathophysiology that predisposes isolated dystonia patients to progressive cognitive impairment such as in PD, we hypothesised that STN DBS would disrupt cognitive-motor dual-task performance to a lesser degree than is seen in neurodegenerative conditions such as PD. This study may help further inform potential clinical expectations following STN DBS for dystonia, and provide better understanding of the role of STN in working memory and attention outside the setting of a neurodegenerative disorder.

\section{METHODS}

We performed a prospective, unblinded, within-participants experiment in which participants were tested in stimulation 'on' and 'off' conditions, both in single-task and dual-task paradigms over a $4.5 \mathrm{~h}$ testing period. Patients who had previously undergone STN DBS surgery for dystonia were enrolled if they were over the age of 18, English speaking and had stable DBS settings for at least 1 month. They were excluded if dystonia was not isolated or if the Montreal Cognitive Assessment battery (MoCA) score was under 22. A power calculation with $n=8$ patients, using a two-sided $\alpha$ of 0.05 with an effect size of $50 \%$ of that found in a similar study ${ }^{28}$ showed a power of 0.921 . Institutional Review Board (IRB) approval was obtained prior to enrolment (UCSF IRB\# 10-00783). Participant data collected during the research visit included standard demographics, symptom duration, age at surgery, years of education, MoCA and preoperative and current BFMDRS and TWSTRS scores.

\section{Testing protocol}

Patients completed fine motor (force maintenance task) and working memory (n-back task) tasks both in isolation (singletask) and simultaneously (dual-task). Three degrees of difficulty for the n-back task (0-back, 1-back, 2-back) were performed in single-task and dual-task paradigms. To minimise bias from practice effects, the order of the n-back task difficulty was randomised and participants underwent three practice trials in each specific testing scenario until performance plateaued before the test trials were recorded. Thirty-second rest periods between each test trial helped to avoid fatigue. The entire testing battery was repeated in the stimulation 'off' state following a $1 \mathrm{~h}$ washout period. Patients were not asked to withhold any oral dystonia medications.

\section{Motor testing: force maintenance test}

This paradigm was adapted from work by Alberts $e t a l^{28}$ in older healthy adults and patients with PD. Fine motor function was assessed with a $6 \mathrm{df}$ force-torque $(\mathrm{F} / \mathrm{T})$ transducer (Mini-40, ATI Industrial Automaation, Garner, North Carolina, USA) measuring the precision grip force (thumb and index finger) of the dominant hand and used force-maintenance as a marker of concentration on a motor task. The F/T transducer was interfaced with a custom LabView program developed by the Alberts et al group and a 21 in computer screen provided a visual representation of the level of force applied to the transducer. Data collected with the LabView program were filtered using a low-pass filter using Woltring's algorithm in novel Matlab analysis programs as described previously. ${ }^{30}$

During motor testing, a target force level was set on the visual display at $20 \%$ of the participant's maximum force and the participant was asked to match their force production on a real-time display as closely as possible to the target force line.
Variability in force tracking was recorded as the relative root mean square error (RRMSE) in order to normalise performance to force amplitude, ${ }^{31}$ such that a lower RRMSE indicated less variability in force tracking. RRMSE was transformed to the log of RRMSE (logRRMSE) for normalisation. Ten such trials were completed for each participant with a $30 \mathrm{~s}$ break and three practice trials between each testing interval. During the dual-task testing where force maintenance is performed simultaneously with the n-back test, five intervals were carried out at each level of difficulty of the n-back test.

\section{Cognitive testing: the $\mathrm{n}$-back test}

Attention to a cognitive task was tested using the working memory ' $n$-back' test, ${ }^{32}$ which requires information encoding, maintenance, updating and output but no comparison or decision-making. A list of randomly generated letters was read and the participant was asked to recall the n-th item back in a sequentially presented list of items. One experimenter read the list of letters $(1 / \mathrm{s})$ while the second experimenter graded the verbal performance. When an error was made or the participant delayed his or her answer, the reading experimenter started a new list of letters. Each trial lasted $30 \mathrm{~s}$ (15-21 letters being read per trial). The test varied in difficulty (0-back, 1-back and 2-back), with the easiest condition (0-back) requiring the participant to repeat the last letter spoken by the examiner. At the '2-back' level, while hearing the sequence 'A, C, F, U, D, L, Z, $\mathrm{K}$...' the participant would repeat ' $\mathrm{A}$ ' after hearing the letter ' $\mathrm{F}$ ', the letter ' $\mathrm{C}$ ' after hearing ' $\mathrm{U}$ ' and so on. Under both single-task and dual-task testing conditions, five $30 \mathrm{~s}$ trials were performed at each level of difficulty (0-back, 1-back and 2-back). The order of the three levels of n-back difficulty was randomised to avoid learning effects as the difficulty increased. Participants were given three $30 \mathrm{~s}$ practice trials at each degree of $n$-back difficulty to ensure that the performance plateaued and all test blocks contained unique sequences of randomised letters. The primary outcome measures for the $n$-back test included the percentage of correct letters repeated and the total number of errors (NE) committed during a $30 \mathrm{~s}$ trial. ${ }^{30}$

\section{Statistical analyses}

Repeated measures analysis of variance (ANOVA) was used to analyse motor and cognitive data. For the cognitive data, a $2 \times 3 \times 2$ (stimulation off vs on) $\times(\mathrm{n}$-back difficulty $\mathrm{n}=0, \mathrm{n}=1$, $\mathrm{n}=2) \times($ task paradigm single-task vs dual-task) experimental design was analysed for each outcome variable (NE and percentage correct). For motor data, a $2 \times 3 \times 2$ (stimulation off vs on $) \times(n$-back difficulty $n=0, n=1, n=2) \times($ task paradigm singletask vs dual-task) experimental design was analysed using the $\log$ transformation of RRMSE, which is a measure of overall variability in force-tracking performance.

In an alternative analysis, performance under dual-task conditions was compared to performance in either task alone by calculating the dual-task loss (DTL) ${ }^{33}$ as the percentage of decrement in motor or cognitive performance during DT conditions relative to performance in the single-task (ST) conditions:

$$
\mathrm{DTL}_{\mathrm{x}}=\left[\left(\text { mean DT }_{\mathrm{x}}-\text { mean ST }_{\mathrm{x}}\right) / \text { mean ST }_{\mathrm{x}}\right] \times 100
$$

where $\mathrm{x}=$ either NE, percentage correct or log RRMSE.

We then compared the mean DTLs to zero with a series of one-sample $t$ tests for each DBS condition controlling for multiple comparisons and ANOVAs were used to compare the DTLs for task difficulties (0-back, 1-back, 2-back) and DBS 
status (on, off). The significance level was set at 0.05. All analyses were conducted using STATA V.12 (College Station, Texas, USA).

To determine if preoperative cognitive status predicted dualtask performance, we correlated preoperative MoCA with postoperative n-back score.

\section{RESULTS}

Baseline clinical data

The clinical characteristics of eight participants are shown in table 1 .

All participants were recruited from a cohort of patients who underwent STN DBS for isolated idiopathic craniocervical dystonia with an average age of 56.8 years and with a mean dystonia duration of $10.5 \pm 3$ years $( \pm S D)$. The average MoCA score $(26.5 \pm 2.1)$ was above the typical cut-off for cognitive impairment (26) in English-speaking persons with at least 12 years of education. Participants had full preoperative and postoperative neuropsychological evaluations (not simultaneous with the dual-task study) and no significant declines in performance were noted. The mean change in dystonia rating scales from the preoperative assessment to the time of dual-task testing was $48 \% \pm 10$ using the BFMDRS-movement score, 52\% \pm 8 for TWSTRS severity subscore and $41 \% \pm 32$ for TWSTRS disability subscore, although a large variability was seen in outcomes across the patients.

\section{Working memory outcomes}

\section{Percentage of correct responses}

On average, participants generally performed well on the n-back task (>85\% correct), suggesting adequate performance. ${ }^{32}$ The repeated measures ANOVA showed a statistically significant worsening of performance, as measured by 'percentage correct', on the n-back test with increasing task difficulty $(F=133.85$, $\mathrm{p}<0.001$ ). However, there was no main effect of task (single vs dual) $(\mathrm{F}=1.06, \mathrm{p}=0.3112)$ or DBS status ('on' vs 'off') $(\mathrm{F}=0.75, \mathrm{p}=0.393)$ on the percentage of correct answers (figure $1 \mathrm{~A})$. The interaction between task difficulty and stimulation also was not significant $(\mathrm{F}=1.40, \mathrm{p}=0.25)$, indicating that stimulation did not create more of a decline in performance as task difficulty increased.
Number of errors

Similarly, there was a statistically significant effect of task difficulty on the $\mathrm{NE}(\mathrm{F}=135.1, \mathrm{p}<0.001)$, owing to a decline in task performance as n-back difficulty increased. There was no effect of task type $(\mathrm{F}=1.89, \mathrm{p}=0.179)$ or stimulation status $(\mathrm{F}=0.58, \mathrm{p}=0.451)$ on the NE (figure $1 \mathrm{~B})$. The interaction between n-back difficulty and stimulation status was again nonsignificant $(\mathrm{F}=2.38, \mathrm{p}=0.094)$.

\section{DTL for n-back}

DTLs for the percentage of correct answers on the n-back were not significantly different from zero using one-sample $t$ tests with either DBS 'on' $\left(\mathrm{DTL}_{0 \text {-back }}=-2.3, \mathrm{p}=0.054 ; \mathrm{DTL}_{1 \text {-back }}=\right.$ $-2.79, \mathrm{p}=0.34 ; \mathrm{DTL}_{2 \text {-back }}=-0.978, \mathrm{p}=0.40$ ) or DBS 'off' $\left(\mathrm{DTL}_{0 \text {-back }}=0.415, \mathrm{p}=0.724 ; \mathrm{DTL}_{1 \text {-back }}=-0.553, \mathrm{p}=0.75\right.$; $\mathrm{DTL}_{2 \text {-back }}=1.92, \mathrm{p}=0.38$ ). However, a repeated measures ANOVA did show a statistically significant effect of stimulation status on DTL $(\mathrm{F}=9.14, \mathrm{p}=0.019)$, indicating that participants saw more of a difference in performance between single-task and dual-task conditions in the DBS 'on' state than the DBS 'off' state (figure 3A). DTLs of the n-back test did not vary based on n-back difficulty $(\mathrm{F}=0.75, \mathrm{p}=0.48)$.

\section{Force-tracking outcomes \\ Log of RRMSE}

The distribution of RRMSE did not meet the normality criteria, and a mathematical transformation ( $\log$ of RRMSE) was used for statistical analysis (figure 2). The model showed a statistically significant effect of task (single-task vs dual-task) on logRRMSE, where the performance was worse during the dual-task condition $(\mathrm{F}=22.38, \mathrm{p}=0.042)$. There was no effect of stimulation status $(F=0.22, p=0.69)$ or $n$-back difficulty $(F=0.03, p=0.97)$ on force-tracking as measured with the logRRMSE. However, the interaction between n-back difficulty and stimulation status was significant $(\mathrm{F}=3.01, \mathrm{p}=0.03)$, indicating that as the $\mathrm{n}$-back task became more difficult, stimulation was more likely to worsen performance on force tracking.

\section{DTL for force-tracking}

There was a non-significant trend towards an effect of stimulation status on DTL when using force maintenance performance as the outcome $(\mathrm{F}=3.8, \mathrm{p}=0.092$; figure $3 \mathrm{~B})$. There was no effect of n-back difficulty on force maintenance DTL $(F=0.34$,

Table 1 Characteristics of patients who underwent bilateral STN DBS for dystonia, including dystonia rating scale change at the time of dual-task testing compared to preoperative scores

\begin{tabular}{|c|c|c|c|c|c|c|c|c|c|}
\hline \multirow[b]{2}{*}{$\begin{array}{l}\text { Participant } \\
\text { number }\end{array}$} & \multirow[b]{2}{*}{$\begin{array}{l}\text { Age at } \\
\text { onset (year) }\end{array}$} & \multirow[b]{2}{*}{ Gender } & \multirow[b]{2}{*}{$\begin{array}{l}\text { Onset of } \\
\text { dystonia }\end{array}$} & \multirow[b]{2}{*}{$\begin{array}{l}\text { Disease } \\
\text { duration (year) }\end{array}$} & \multirow[b]{2}{*}{$\begin{array}{l}\text { Age at } \\
\text { surgery (year) }\end{array}$} & \multirow[b]{2}{*}{$\begin{array}{l}\text { Education } \\
\text { (year) }\end{array}$} & \multirow[b]{2}{*}{$\begin{array}{l}\text { Baseline } \\
\text { MoCA }\end{array}$} & \multicolumn{2}{|c|}{ Percentage of improvement* } \\
\hline & & & & & & & & $\begin{array}{l}\text { BFMDRS } \\
\text { movement }\end{array}$ & $\begin{array}{l}\text { TWSTRS } \\
\text { severity }\end{array}$ \\
\hline 1 & 52 & $M$ & Neck & 11 & 63 & 13 & 24 & 51 & 31 \\
\hline 2 & 58 & $\mathrm{~F}$ & $\begin{array}{l}\text { Mouth, neck, } \\
\text { left hand }\end{array}$ & 10 & 68 & 15 & 27 & 70 & 76 \\
\hline 3 & 49 & $\mathrm{~F}$ & Eyes & 6 & 55 & 12 & 30 & 85 & 69 \\
\hline 4 & 48 & $M$ & Neck & 16 & 64 & 12 & 25 & 36 & 64 \\
\hline 5 & 44 & $\mathrm{~F}$ & Neck & 4 & 48 & 12 & 28 & 56 & 82 \\
\hline 6 & 63 & M & Neck & 3 & 66 & 15 & 26 & 83 & 70 \\
\hline 7 & 37 & $\mathrm{~F}$ & Neck & 7 & 45 & 13 & 28 & 5 & 20 \\
\hline 8 & 18 & $M$ & Right hand & 27 & 45 & 13 & 24 & 0 & 8 \\
\hline Mean \pm SE & $46.5 \pm 5$ & & & $10.5 \pm 3$ & $56.8 \pm 3$ & $13.3 \pm 0$ & $26.5 \pm 1$ & $48.3 \pm 12$ & $52.4 \pm 10$ \\
\hline
\end{tabular}


Figure 1 Results of n-back test in single-task and dual-task conditions, each in the subthalamic nucleus stimulation 'on' and 'off' states, and each over three levels of $n$-back difficulty. (A) Percentage of correct responses in $30 \mathrm{~s}$ of $n$-back, five trials averaged for each condition. (B) Number of errors in $30 \mathrm{~s}$ of $n$-back, five trials averaged for each condition. Error bars representSE.
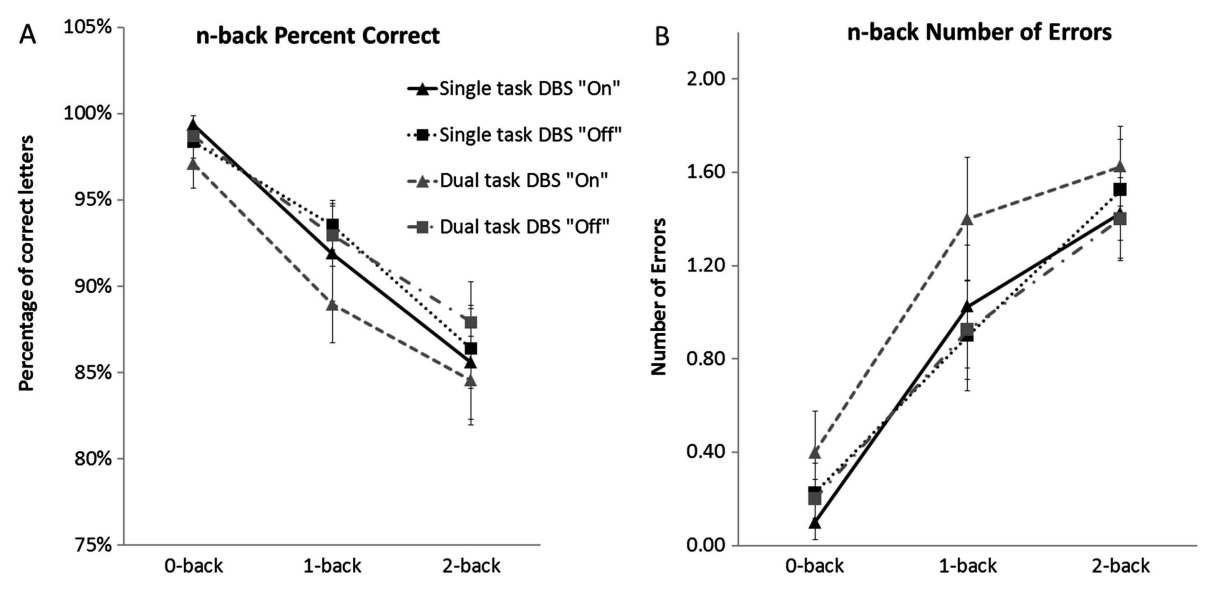

$p=0.71$ ). One participant reported difficulty performing the motor task in the DBS 'off' state due to the worsened retrocollis, but his individual scores still showed a decrement in the DBS 'on' state.

\section{DISCUSSION}

We found no significant effect of subthalamic stimulation (on vs off) on n-back or force-tracking performance in patients with dystonia. There was no main effect of task type (single-task vs dual-task) on either the NE or percentage correct during the n-back task, and the interaction between n-back difficulty and task type was non-significant. The degree of n-back difficulty did have an effect on n-back performance, validating that the degree of $n$-back difficulty increases the cognitive load, but this

\section{Force maintenance}

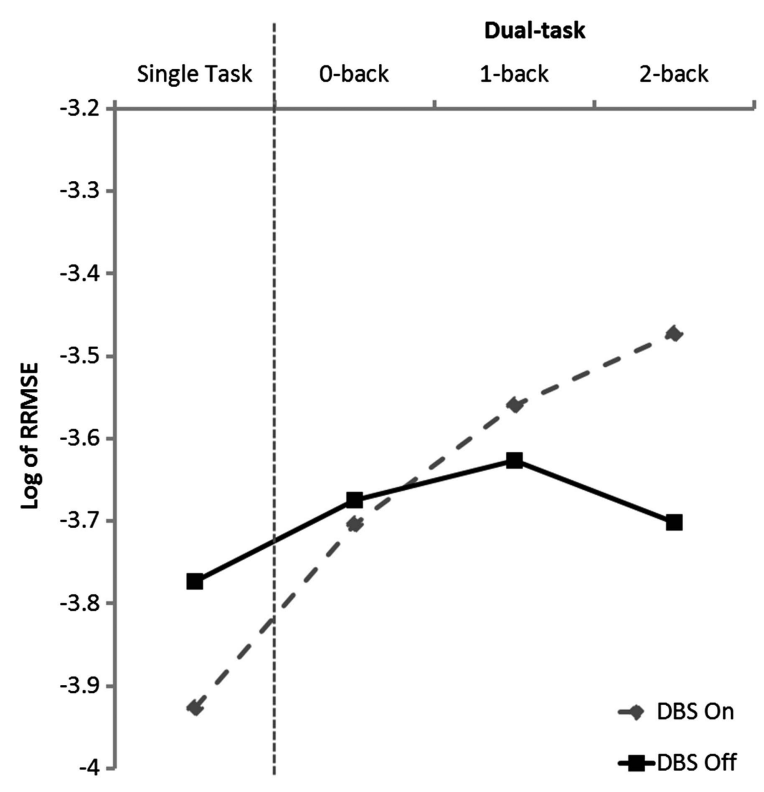

Figure 2 Results of force maintenance test in single-task and dual-task conditions, each in the subthalamic nucleus stimulation 'on' and 'off' states. Dual-task testing was performed in three levels of difficulty, with five trials in each specific set of conditions. The outcome is the log transformation of the relative root mean square error (RRMSE), a measure of variability in force tracking performance, where a more negative logRRMSE indicates better performance on the motor task. relationship did not vary depending on the stimulation status or task type (single vs dual).

By comparison, in patients with PD, Alberts et al showed an absolute worsening by $5.5 \%$ of correct responses in the n-back test during single-task conditions with DBS 'off' compared to 'bilateral on', with a worsening to an absolute drop of $9 \%$ between DBS 'off' and 'bilateral on' under the most difficult (2-back) dual-task conditions. These differences were statistically significant. At the 2-back level of difficulty, we saw only a drop from $86.4 \%$ to $85.6 \%$ correct ( 0.8 difference) on the n-back under single-task conditions and a drop from $87.9 \%$ to $84.5 \%$ correct (difference of 3.4) under dual-task conditions. Our study was powered to find at least a difference of $4 \%$ correct between stimulation off and bilateral stimulation on conditions at the 2-back level of difficulty, which was less than half of that seen in the patients with PD studied in the Alberts et al experiment.

Using this type of analysis, bilateral STN DBS does not appear to have a substantial effect on working memory or attention to motor function either in single-task or dual-task conditions in patients with dystonia.

\section{Interpretation of DTL}

DTL represents another way to conceptualise the difference between performance in single-task and dual-task testing paradigms when other variables are held constant. DTLs for the percentage of correct $n$-back answers in patients with PD showed a $8 \%$ drop in performance at the 1-back level of difficulty and approximately an 5\% worsening at the 2-back level of difficulty, ${ }^{28}$ whereas our participants with dystonia saw only a $2.8 \%$ and $1 \%$ worsening at the 1 -back and 2-back levels of difficulty, respectively, under dual-task conditions with stimulation on. DTLs for the motor outcome, log of RRMSE, showed a similar trend but also had similarly small absolute DTL sizes (1\% loss for DBS on at the 2-back level of difficulty), with no effect of difficulty on DTL.

Though a significant effect of stimulation on DTL for the n-back outcomes was found, the DTLs themselves are not significantly different from 0 and represent much smaller losses in performance than those reported in $\mathrm{PD}$, making this less likely to be a clinically significant effect.

\section{Comparison with PD}

The lack of a more robust effect of stimulation on dual-task performance, as seen with PD, could be due to differences in age of the study populations, perhaps differences in surgical 
Figure 3 Results of dual-task loss (DTL) calculations, representing the per cent change in performance on either (A) n-back or (B) force-maintenance when going from single-task to dual-task. Results are shown for both stimulation states and all levels of n-back difficulty.

(A) DTLs based on percentage of correct $n$-back answers. (B) DTLs based on log of RRMSE. Negative DTLS indicate worsening of scores under dual-task conditions relative to single-task conditions (baseline).

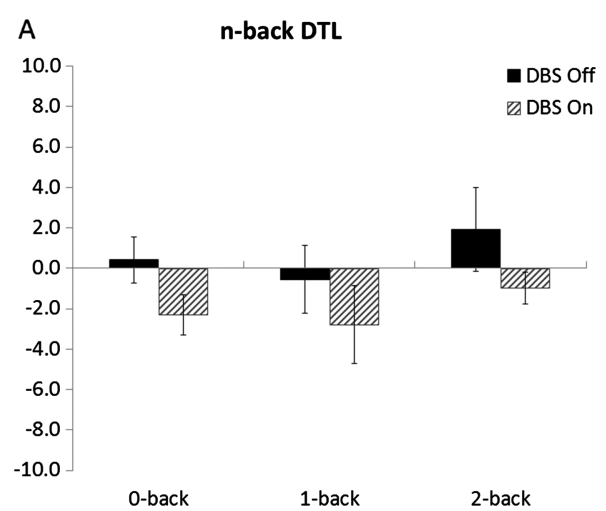

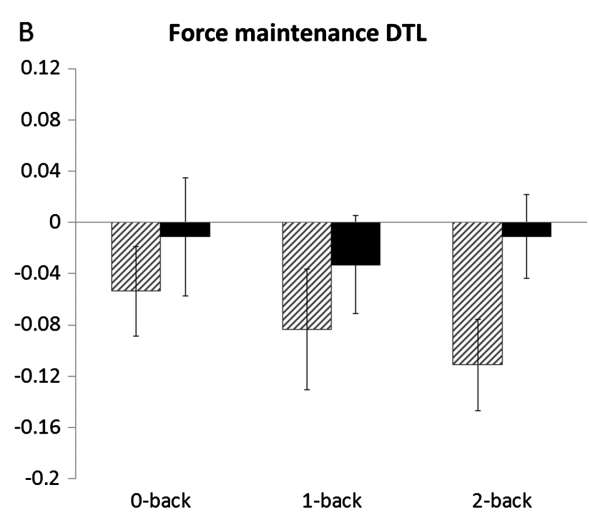

techniques or lead trajectory, or due to differences in underlying disease pathophysiology which predispose patients to stimulation-induced changes in attention or working memory. Dual-task performance is generally worse than single-task performance even in healthy adults, ${ }^{34}$ a trend which worsens even in healthy participants with age. ${ }^{33}$ However, the average age of our study cohort (56.8 years) was similar to that of the patients with PD used by the Cleveland group (56.5 years), so differences in dual-task performance are most likely not explained by age. Caudate nucleus lead penetration has been reported with worsening cognition after STN DBS ${ }^{35}$ The method for lead implantation was similar across cohorts, but we are not certain how often the caudate nucleus was traversed in the PD cohort. In our study, the DBS lead grazed the head of the caudate in three patients on one side, and their DTLs were not significantly different from those of other participants with dystonia.

Cognitive dysfunction often exists to some degree even in early or previously undiagnosed PD. In one population-based case-control study, early patients with PD (35\% previously undiagnosed) performed significantly worse on the 37-item MMSE, verbal fluency and delayed recall items compared with age-matched controls. ${ }^{36}$ Imaging studies including patients with PD across the spectrum of cognitive impairment have correlated executive function, attention and memory with cortical atrophy ${ }^{37}$ and a loss of microstructural white matter integrity as early markers for this cognitive impairment. ${ }^{38}$ Cognitive testing and anatomical research suggest that even when motor symptoms predominate, early changes in cognition are often present.

Compared to age-matched controls, gait worsens in patients with PD with cognitive loading, ${ }^{24} 27$ but Panyakaew et al ${ }^{39}$ showed that even patients with early PD (Hoehn and Yahr stage 1) had a worsening of several measures of gait function when dual-tasking. Most recently, it was shown that with cognitive loading in non-demented patients with PD (mean MMSE =29.1), verbal fluency was correlated with disability and impairment under dual-task conditions. ${ }^{40}$ The previously discussed Alberts et al, ${ }^{28}$ paper suggests that bilateral STN DBS may worsen this baseline deficit in PD patients.

The lack of structural changes and the less reproducible reports of cognitive dysfunction ${ }^{3} 46$ in patients with isolated dystonia could explain the lack of a substantial effect of STN stimulation on dual task performance, at least to the degree seen in PD. Whereas the progressive neurodegeneration and nigrostriatal dopaminergic denervation seen in PD creates a more susceptible substrate for stimulation-induced cognitive changes, the biochemical and/or physiological changes in circuit function seen in dystonia may still allow for utilisation of 'cognitive reserve' even with the addition of another stressor such as STN stimulation. While the small sample size limited our ability to consider baseline predictive variables in the analysis, preoperative MoCA tended to correlate with dual-task performance under the most difficult testing scenario $\left(R_{2}=0.314, p>0.05\right)$ (see online supplementary figure S1).

This study has several limitations. The sample size was small, though the within-participant design allowed for control of inter-participant variation and we met the enrolment goal for the power calculation, which estimated that eight participants would be needed to show at least half the n-back performance decrement seen in patients with PD during stimulation. We also lacked direct comparison with a PD cohort to confirm that there was a disease-dependent effect of STN DBS on dual-task function. However, our protocol followed the same methodology used by Albert and colleagues, and therefore it is reasonable to compare the findings of the two studies. Experimenters and participants were not blinded to stimulation status, though the motor task was automated, and thus the outcome could not be biased by the experimenters. Owing to the worsening of dystonia in the stimulation 'off' state, blinding the participants to stimulation status was not possible. The sequence of 'on' and 'off' stimulation was not randomised to minimise duration of the testing session, but the order of task type and difficulty was randomised and participants plateaued through practice sessions to minimise a learning bias. Performance on the n-back test showed a small NE across trials, and more variability in errors might have increased our ability to detect differences between groups.

Results from our study are reassuring for the use of STN DBS in patients with isolated dystonia with regard to its effect on dual-task cognitive-motor function, and suggest that assumptions about stimulation-induced cognitive side effects may not be generalisable across diseases even when the same brain structure is targeted.

Acknowledgements The authors would like to thank all participants and Robin Taylor for her help with IRB processing.

\section{Collaborators Salman Qasim.}

Contributors KAM contributed to the study design, data acquisition, data analysis, drafting and revision of the manuscript, and is the guarantor of the paper. LCM contributed to the data acquisition, data analysis and revision of the manuscript. MSL contributed to the data analysis, drafting and revision of the manuscript and gave final approval for publication. RR contributed to the data acquisition, data analysis and revision of the manuscript. IEA contributed to the study design, data analysis and drafting of the manuscript. CAR contributed to the study design, data analysis and revision of the manuscript. JLA contributed to the study design, data analysis and revision of the manuscript. PAS contributed to the study design, drafting and revision of the manuscript and gave final approval for publication. JLO contributed to the study design, data collection, data analysis, revision of the manuscript and gave final approval for publication.

Funding This project was supported by NIH/NCRR UCSF-CTSI Grant Number UL1RR024131. 
Competing interests None.

Ethics approval University of California San Francisco Committee on Human Research.

Provenance and peer review Not commissioned; externally peer reviewed.

Data sharing statement Additional data include raw force maintenance measurements, including force data from the six degrees of freedom force-torque transducer in all testing conditions.

Open Access This is an Open Access article distributed in accordance with the Creative Commons Attribution Non Commercial (CC BY-NC 3.0) license, which permits others to distribute, remix, adapt, build upon this work non-commercially, and license their derivative works on different terms, provided the original work is properly cited and the use is non-commercial. See: http://creativecommons.org/ licenses/by-nc/3.0/

\section{REFERENCES}

1 Albanese A, Bhatia K, Bressman SB, et al. Phenomenology and classification of dystonia: a consensus update. Mov Disord 2013;28:863-73.

2 Hendrix CM, Vitek JL. Toward a network model of dystonia. Ann N Y Acad Sci 2012;1265:46-55

3 Pillon B, Ardouin C, Dujardin K, et al. Preservation of cognitive function in dystonia treated by pallidal stimulation. Neurology 2006;66:1556-8.

4 Bugalho P, Correa B, Guimaraes J, et al. Set-shifting and behavioral dysfunction in primary focal dystonia. Mov Disord 2008:23:200-6.

5 Jahanshahi M, Rowe J, Fuller R. Cognitive executive function in dystonia. Mov Disord 2003:18:1470-81.

6 Balas M, Peretz C, Badarny S, et al. Neuropsychological profile of DYT1 dystonia. Mov Disord 2006:21:2073-7.

7 Stamelou M, Edwards MJ, Hallett M, et al. The non-motor syndrome of primary dystonia: clinical and pathophysiological implications. Brain 2012;135:1668-81.

8 Cif $\mathrm{L}$, El Fertit $\mathrm{H}$, Vayssiere $\mathrm{N}$, et al. Treatment of dystonic syndromes by chronic electrical stimulation of the internal globus pallidus. J Neurosurg Sci 2003;47:52-5.

9 Vidailhet M, Vercueil L, Houeto JL, et al. Bilateral, pallidal, deep-brain stimulation in primary generalised dystonia: a prospective 3 year follow-up study. Lancet Neurol 2007:6:223-9.

10 Volkmann J, Wolters A, Kupsch A, et al. Pallidal deep brain stimulation in patients with primary generalised or segmental dystonia: 5-year follow-up of a randomised trial. Lancet Neurol 2012;11:1029-38.

11 Ostrem JL, Marks WJ Jr, Volz MM, et al. Pallidal deep brain stimulation in patients with cranial-cervical dystonia (Meige syndrome). Mov Disord 2007;22:1885-91.

12 Walsh RA, Sidiropoulos C, Lozano AM, et al. Bilateral pallidal stimulation in cervica dystonia: blinded evidence of benefit beyond 5 years. Brain 2013;136(Pt 3):761-9.

13 Ostrem JL, Racine CA, Glass GA, et al. Subthalamic nucleus deep brain stimulation in primary cervical dystonia. Neurology 2011;76:870-8.

14 Kleiner-Fisman G, Liang GS, Moberg PJ, et al. Subthalamic nucleus deep brain stimulation for severe idiopathic dystonia: impact on severity, neuropsychological status, and quality of life. J Neurosurg 2007;107:29-36.

15 Sun $B$, Chen $S$, Zhan $S$, et al. Subthalamic nucleus stimulation for primary dystonia and tardive dystonia. Acta Neurochir Supp/ 2007;97:207-14.

16 Schrader C, Capelle HH, Kinfe TM, et al. GPi-DBS may induce a hypokinetic gait disorder with freezing of gait in patients with dystonia. Neurology 2011; 77:483-8.

17 Zauber SE, Watson N, Comella CL, et al. Stimulation-induced parkinsonism after posteroventral deep brain stimulation of the globus pallidus internus for craniocervical dystonia. J Neurosurg 2009;110:229-33.

18 Berman BD, Starr PA, Marks WJ Jr, et al. Induction of bradykinesia with pallidal deep brain stimulation in patients with cranial-cervical dystonia. Stereotact Funct Neurosurg 2009;87:37-44.
19 Pauls KA, Hammesfahr $\mathrm{S}$, Moro $\mathrm{E}$, et al. Deep brain stimulation in the ventrolateral thalamus/subthalamic area in dystonia with head tremor. Mov Disord 2014;29:953-9.

20 Parsons TD, Rogers SA, Braaten AJ, et al. Cognitive sequelae of subthalamic nucleus deep brain stimulation in Parkinson's disease: a meta-analysis. Lancet Neurol 2006:5:578-88.

21 Witt K, Daniels C, Reiff J, et al. Neuropsychological and psychiatric changes after deep brain stimulation for Parkinson's disease: a randomised, multicentre study. Lancet Neurol 2008;7:605-14.

22 Follett KA, Weaver FM, Stern M, et al. Pallidal versus subthalamic deep-brain stimulation for Parkinson's disease. N Engl J Med 2010;362:2077-91.

23 Woollacott M, Shumway-Cook A. Attention and the control of posture and gait: a review of an emerging area of research. Gait Posture 2002;16:1-14.

24 Yogev G, Giladi N, Peretz C, et al. Dual tasking, gait rhythmicity, and Parkinson's disease: which aspects of gait are attention demanding? Eur J Neurosci 2005;22:1248-56.

25 Pillon B, Ardouin C, Damier P, et al. Neuropsychological changes between "off" and "on" STN or GPi stimulation in Parkinson's disease. Neurology 2000;55:411-18.

26 Rivaud-Pechoux S, Vermersch Al, Gaymard B, et al. Improvement of memory guided saccades in parkinsonian patients by high frequency subthalamic nucleus stimulation. J Neurol Neurosurg Psychiatry 2000;68:381-4.

27 Rochester $L$, Hetherington $V$, Jones $D$, et al. Attending to the task: interference effects of functional tasks on walking in Parkinsons disease and the roles of cognition, depression, fatigue, and balance. Arch Phys Med Rehabil 2004;85:1578-85.

28 Alberts JL, Voelcker-Rehage C, Hallahan K, et al. Bilateral subthalamic stimulation impairs cognitive-motor performance in Parkinson's disease patients. Brain 2008;131:3348-60.

29 Halbig TD, Gruber D, Kopp UA, et al. Pallidal stimulation in dystonia: effects on cognition, mood, and quality of life. J Neurol Neurosurg Psychiatry 2005:76:1713-16.

30 Voelcker-Rehage C, Stronge AJ, Alberts JL. Age-related differences in working memory and force control under dual-task conditions. Neuropsychol Dev Cogn B Aging Neuropsychol Cogn 2006;13:366-84.

31 Kriz G, Hermsdorfer J, Marquardt C, et al. Feedback-based training of grip force control in patients with brain damage. Arch Phys Med Rehabil 1995 76:653-9.

32 Dobbs AR, Rule BG. Adult age differences in working memory. Psychol Aging 1989;4:500-3.

33 Lindenberger U, Marsiske M, Baltes PB. Memorizing while walking: increase in dual-task costs from young adulthood to old age. Psychol Aging 2000;15:417-36.

34 Grabiner MD, Troy KL. Attention demanding tasks during treadmill walking reduce step width variability in young adults. J Neuroeng Rehabil 2005;2:25.

35 Witt K, Granert O, Daniels C, et al. Relation of lead trajectory and electrode position to neuropsychological outcomes of subthalamic neurostimulation in Parkinson's disease: results from a randomized trial. Brain 2013;136:2109-19.

36 Benito-Leon J, Louis ED, Posada IJ, et al. Population-based case-control study of cognitive function in early Parkinson's disease (NEDICES). J Neurol Sci 2011:310:176-82.

37 Biundo R, Calabrese M, Weis $L$, et al. Anatomical correlates of cognitive functions in early Parkinson's disease patients. PLOS ONE 2013;8:e64222.

38 Melzer TR, Watts R, MacAskill MR, et al. White matter microstructure deteriorates across cognitive stages in Parkinson disease. Neurology 2013;80:1841-9.

39 Panyakaew P, Bhidayasiri R. The spectrum of preclinical gait disorders in early Parkinson's disease: subclinical gait abnormalities and compensatory mechanisms revealed with dual tasking. J Neural Transm 2013;120:1665-72.

40 Fuller RL, Van Winkle EP, Anderson KE, et al. Dual task performance in Parkinson's disease: a sensitive predictor of impairment and disability. Parkinsonism Relat Disord 2013;19:325-8. 